\title{
Splenic peliosis associated with rupture in a renal transplant patient
}

\author{
M. A. Parsons \\ M.B., Ch.B. \\ M. Platts \\ M.D., M.R.C.P.
}

\author{
D. Slater \\ B.Med.Sci., M.R.C.Path. \\ M. Fox \\ Ch.M., F.R.C.S.
}

The Royal Hallamshire Hospital, Sheffield

\begin{abstract}
Summary
A patient is described who, 2 years after renal transplantation, presented as an acute abdominal emergency due to a ruptured spleen. Histologically this displayed peliosis.

\section{Introduction}

Peliosis is an uncommon entity consisting of bloodfilled cavities found most frequently in the liver (peliosis hepatis), although its rare occurrence in the spleen has been described. Originally splenic peliosis was considered a histological curiosity with no clinical significance. Recently, however, 2 cases of fatal haemoperitoneum have been reported following rupture of splenic peliotic cysts (Taxy, 1978; Benjamin and Shunk, 1978).

The authors report an additional case of splenic peliosis associated with non-fatal rupture in a renal transplant patient.
\end{abstract}

\section{Case report}

In 1967, a 21-year-old man presented with chronic renal failure due to chronic glomerulonephritis. After initial peritoneal and haemodialysis in hospital, home dialysis was commenced in 1969. A successful cadaver renal transplant was performed in May 1972. Weekly intramuscular injections of $250 \mathrm{mg}$ testosterone were begun in June 1971 for anaemia $(6 \mathrm{~g} / \mathrm{dl})$ but were stopped after transplantation. He was maintained on immunosuppressive therapy (azathioprine $100 \mathrm{mg}$ and prednisolone $12.5 \mathrm{mg}$ daily) and remained well until October 1974. He then presented with a sudden onset of pain in the les upper abdominal quadrant with radiation to the shoulder. A mass developed in the left uppes quadrant and his haemoglobin fell from 14 to. $11 \mathrm{~g} / \mathrm{dl}$. The clinical impression was that of ruptured spleen. There had been no history of trauma, and tests for infectious mononucleosis were

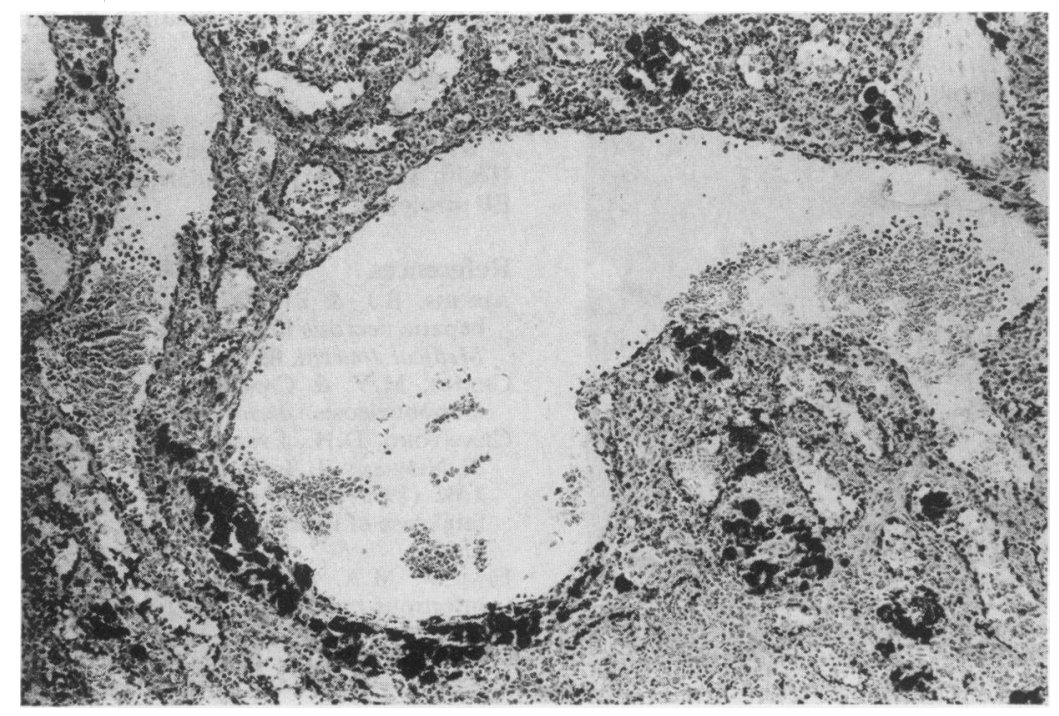

FIG. 1. The spleen showing blood-filled peliotic cavities and dilated sinusoids. Darkly staining haemosiderin deposits can be seen at the edge of one large cavity (Perls stain, $\times 80$ ).

0032-5473/80/0700-0796 $\$ 02.00$ (C) 1980 The Fellowship of Postgraduate Medicine 
negative. The spleen was found to be surrounded by a large haematoma at laparotomy and was removed. The liver showed no macroscopic abnormality and 'liver function tests' have remained normal. Subsequent progress has been uneventful.

The spleen weighed $210 \mathrm{~g}$ and the distal end showed a large ruptured subcapsular haematoma. The red pulp contained scattered blood-filled cavities (up to $3 \mathrm{~mm}$ diameter), adjacent to the haematoma and in subcapsular areas (Fig. 1). The larger cavities appeared continuous with ectatic sinusoids but littoral cells were not always demonstrable. These appearances were considered to be those of splenic peliosis. In addition, plentiful haemosiderin-containing macrophages were present in the red pulp and in places lined the sinusoids and peliotic cavities. No increase in fibrous tissue could be demonstrated, and the white pulp was mildly depleted. The splenic artery and vein were normal.

\section{Discussion}

Splenic peliosis was initially described in patients with debilitating diseases such as tuberculosis and malignancy. Peliosis hepatis was also invariably present and recent reports confirm such an association (Taxy, 1978; Benjamin and Shunk, 1978; Lacson, Berman and Neiman, 1979). Taxy (1978) has reported 2 cases of splenic peliosis without hepatic involvement and it is likely that the present case conforms to isolated splenic peliosis, although a liver biopsy was not performed.
Suggested aetiological factors in the development of peliosis have included anabolic steroids and azathioprine (Taxy, 1978; Benjamin and Shunk, 1978; Lacson, et al., 1979; Degott et al., 1978) and it is perhaps relevant that the patient had received both these drugs. An interesting finding in this case was the heavy deposition of haemosiderin in the spleen. This feature is frequently seen in renal dialysis and transplant patients, and is currently under investigation. It is possible that the haemosiderin interfered with vascular drainage and gave rise to phlebectatic peliotic cavities as described by Yanoff and Rawson (1964). From the distribution of the lesions it would appear probable that the haemorrhage originated from a ruptured peliotic cavity. This complication should be considered in renal transplant patients.

\section{References}

Benjamin, D.R. \& Shunk, B. (1978) A fatal case of peliosis of the liver and spleen. American Journal of Diseases of Children, 132, 207.

Degott, C., Rueff, B., Kreis, H., Duboust, A., Potel, Fiona \& Benhamou, J.P. (1978) Peliosis hepatis in recipients of renal transplants. Gut, 19, 748.

LaCson, A., Berman, L.D. \& Neiman, R.S. (1979) Peliosis of the spleen. American Journal of Clinical Pathology, 71, 586.

TAXY, J.B. (1978) A morphologic curiosity becomes an iatrogenic problem. Human Pathology, 9, 331.

Yanoff, M. \& Rawson, A.J. (1964) Peliosis hepatis. An anatomic study with demonstration of two varieties. Archives of Pathology, 77, 159. 